\title{
BMJ Open TRANSCOV cohort protocol: an epidemiological study assessing the impact of critically ill COVID-19 patients long distance transfers between intensive care units
}

Olivier Grimaud (D) , ${ }^{1}$ Sahar Bayat, ${ }^{1}$ Anne Renault, ${ }^{2}$ Eric Maury, ${ }^{3}$ Julien Pottecher, ${ }^{4}$ Manuel Dolz, ${ }^{5}$ François Braun, ${ }^{6}$ Christophe Fermanian, ${ }^{1}$ Agnès Ricard-Hibon, ${ }^{7}$ Nathalie Prieto, ${ }^{8}$ Jean-Marc Philippe, ${ }^{9}$ Emmanuelle Leray ${ }^{10}$

To cite: Grimaud 0 , Bayat S, Renault A, et al. TRANSCOV cohort protocol: an epidemiological study assessing the impact of critically ill COVID-19 patients long distance transfers between intensive care units. BMJ Open 2021;11:e054774. doi:10.1136/ bmjopen-2021-054774

- Prepublication history for this paper is available online. To view these files, please visit the journal online (http://dx.doi. org/10.1136/bmjopen-2021054774).

Received 28 June 2021 Accepted 30 September 2021

Check for updates

(C) Author(s) (or their employer(s)) 2021. Re-use permitted under CC BY-NC. No commercial re-use. See rights and permissions. Published by BMJ.

For numbered affiliations see end of article.

Correspondence to

Dr Olivier Grimaud;

olivier.grimaud@ehesp.fr

\section{ABSTRACT}

Introduction During spring 2020, four regions of France faced a surge of severe COVID-19 patients which threatened to overflow local intensive care units (ICU) capacities. As an emergency response, between 13 March 2020 and 10 April 2020, an estimated 661 patients were transferred from overcrowded ICUs to eight other French regions and four neighbouring countries. The intensity, geographical spread and the diversity of vectors used are unprecedented. The study aims at assessing the impact of these inter-ICU transfers on the short-term and medium-term physical and psychological outcomes in this population of severe COVID-19 patients.

Methods and analysis The TRANSCOV cohort is a multicentre observational retrospective study. All transferred patients between ICUs outside the origin region will be invited to take part. For each transfer, up to four control patients will be selected among those admitted in the same ICU during the same period $( \pm 4$ days of transfer date). Clinical data will be extracted from medical records and will include haemodynamic and respiratory parameters, as well as clinical severity scores before, during and after transfer. Data linkage with medicoadministrative data will enrich the clinical database and allow follow-up up to 1 year after initial admission. Ethics and dissemination The study has been approved by the French Ethics and Scientific Committee on the 16 July 2020 (file no. 2046524). The results will be disseminated via publication of scientific articles and communications in national and international conferences. Trial registration number $20 \mathrm{CO} 015 \mathrm{CZ}$.

\section{INTRODUCTION}

Faced with the COVID-19 epidemic, hospitals located in areas with high circulation of the virus were exposed to an influx of severe patients, which threatened to exceed intensive care unit (ICU) capacities. At the climax of the first epidemic wave in France in April 2020, more than 32000 COVID-19 patients were hospitalised. Over the same
Strengths and limitations of this study

- The study population of the TRANSCOV cohort concerns all intensive care unit COVID-19 patients transferred outside the boundaries of French regions during spring 2020.

- For each transferred patient, up to four control patients will be selected.

- Combining clinical and medicoadministrative data, the TRANSCOV cohort will provide ample opportunities to explore in details short-term and long-term consequences of the transfers.

- Ensuring participation of all centres is the main challenge for the study.

period, more than 7000 patients were treated in ICU (cf dashboard). ${ }^{1}$ Several organisational responses have been made to meet this exceptional demand, including a mechanism for transferring severe patients to ICU located in spared neighbouring regions and countries where the pressure on ICU beds was lower. Thus, between 13 March 2020 and 10 April 2020, an estimated 661 patients were transferred from four regions (Grand Est, Ile de France, Bourgogne-FrancheComté and Corse) to eight French regions (Bretagne, Normandie, Pays de Loire, Centre Val de Loire, Nouvelle Aquitaine, AuvergneRhône-Alpes, Occitanie and Provence Alpes Côte d'Azur) and four countries (Germany, Switzerland, Luxembourg, Austria). Interhospital transfer of critically ill patients is a common and long-established procedure. The wave of COVID-19 patients transfers in spring 2020 is however a unique experience on several accounts: first, the intensity of transfers (ie, high volume in a short time); second, the fact that patients were 
transferred outside regional boundaries (later referred to as long-distance transfers); third, the variety of transport means (vectors) used, including road ambulances, helicopters, planes, boats and high-speed trains. Organising these distant transfers in a context of public health emergency required innovations and advanced collaboration between hospitals teams and health authorities. At the request of the French Health Ministry in April 2020, a team of researchers from the French School of Public Health designed a multidisciplinary 'TRANSCOV' project aiming at drawing all knowledge from this experience of long-distance transfers. TRANSCOV has several components including a qualitative investigation looking at the organisation of the transfers, an economic evaluation component comparing costs of transport modalities and an epidemiological assessment of patients' outcomes. This paper presents the protocol of the latter component, namely the TRANSCOV cohort.

The scientific literature on the clinical impact of transferring patients to intensive care is limited and mainly concerns the transfer of patients from conventional wards or general ICUs to more specialised ones. ${ }^{2}$ With a few exceptions, ${ }^{3}$ it does not provide a comparison of clinical outcomes between transferred versus non-transferred patients, or within the transferred group, between modalities of transport. This also applies to recently published studies providing nevertheless useful descriptive analysis of COVID-19 patients' air transfers in spring $2020 .{ }^{45}$ The TRANSCOV cohort intends to explore these questions as well as to examine the potential effects on patient health of the transfers' duration, medical or material offloads, or the occurrence of adverse events during the transfer. The underlying assumption is that the patients chosen for these often long-distance extraregional transfers were stable enough to withstand the additional stress of a prolonged medical evacuation.

\section{Study objective}

The 'TRANSCOV cohort' aims at measuring physical and psychological impact that inter ICU long-distance transfer might have had on COVID-19 patients. The primary outcomes of the study are short (end of ICU stay), medium (30 days) and long term (up to 1 year) survival. A number of secondary outcomes will be explored related to physiological and care pathways characteristics. More specifically, the study intends to:

1. Describe and compare the clinical characteristics that distinguish patients who were transferred from those who were not.

2. Compare the clinical characteristics of the transferred patients according to the vectors (ambulance, plane, helicopter, boat, train) used.

3. Describe the immediate impact of transfers on the haemodynamic and respiratory parameters of patients, depending on the vectors used.

4. Compare the use of hospital and ambulatory healthcare resources for transferred versus non-transferred patients in the year following hospitalisation in intensive care.

5. Study if and to what extent the impact of the transfer varied according to the characteristics of the patients (eg, age, sex, severity) and of the transfer (eg, vector, duration).

As part of the TRANSCOV project, a distinct component will focus on the psychological impact of distant transfers, for which a specific protocol is being devised.

\section{METHODS AND ANALYSIS \\ Design}

The TRANSCOV cohort uses an observational retrospective design. The study population is composed of a first group of patients who have been exposed to distant transfer and of a group of control ICU patients. The transferred group includes all severe COVID-19 patients admitted in a French ICU ward and then transferred between 13 March 2020 and 10 April 2020 to another ICU located outside the region of the initial ICU stay. Available information indicates that around 661 patients were transferred distantly during the study period from 80 'origin hospitals' towards 150 'destination hospitals'. The control group includes patients selected out of those whose entire ICU care has taken place in one of the origin hospitals. Patients previously admitted from another ICU, or later transferred to another ICU within the region are therefore excluded from the control group.

\section{Sample size}

Given the variety of settings, vectors and likely clinical characteristics, we decided to aim for the enrolment of all patients transferred during the study period thus enabling a detailed description of the whole event. We aim for the enrolment of up to four control patients for each transfer in order to maximise statistical power while keeping costs of data collection manageable. ${ }^{6}$ Between March and April 2020 in France, in-hospital mortality of COVID-19 patients admitted in ICU and with mechanical ventilation has been estimated at $32 \%{ }^{7}$ Given this estimation and assuming $80 \%$ participation in both transfer and control groups, statistical power calculations for the primary outcomes of mortality are as follows: $99 \%$ for $10 \%$ difference in mortality, and respectively $89 \%$ and $57 \%$ for differences of $7.5 \%$ and $5 \%$.

\section{Selection of control patients}

Each origin hospital will select up to four control patients using the following procedure:

1. Identify the ICU admission date and time of the first transferred patient.

2. Identify the group of non-transferred COVID-19 patients admitted to ICU within a range of 4 days before and after this date.

3 . If the number of this group is between 1 and 4, select all patients as control; if the number exceeds 4 patients, select the 4 whose dates and time of admission 


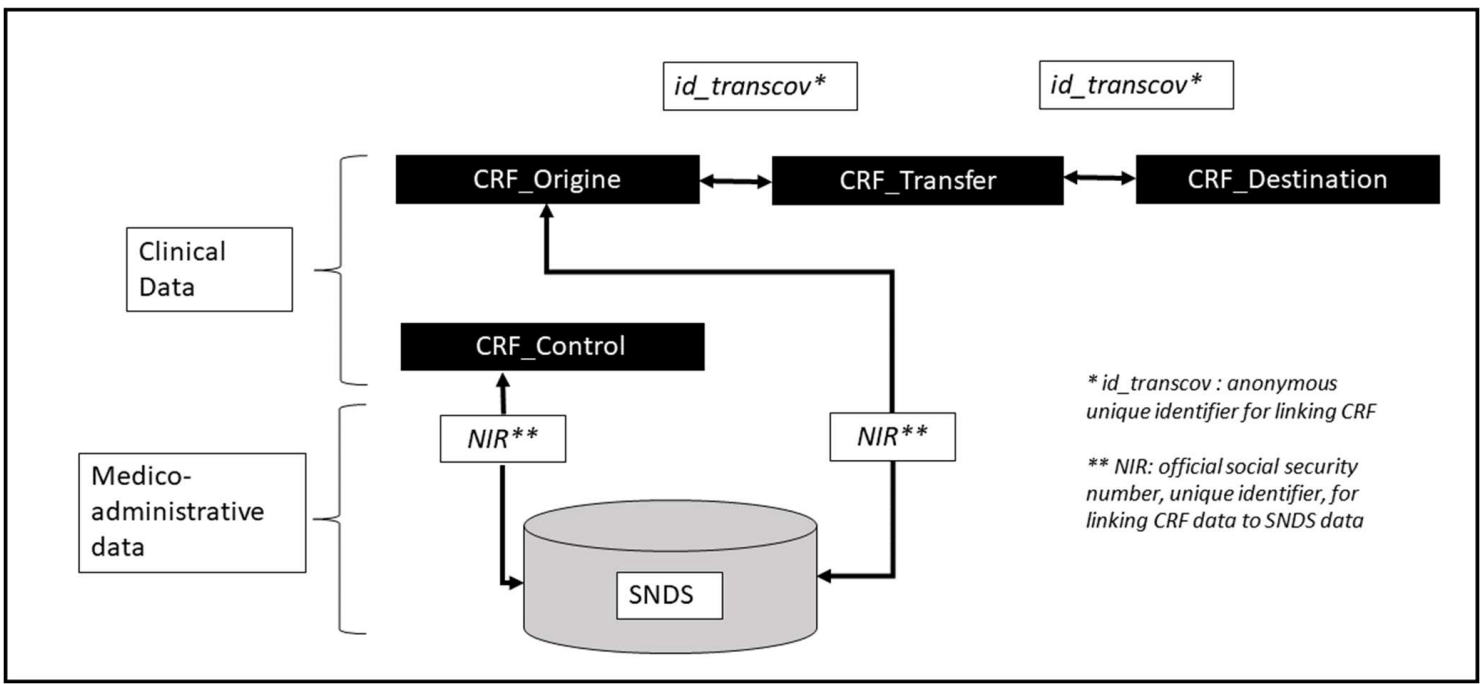

Figure 1 The TRANSCOV cohort database-data sources and linkage. CRF, case report forms; NIR, numéro d'identification au répertoire; SNDS, Système National des Données de Santé.

are the closest chronologically to those of the transferred patient.

4. Repeat steps 1-3 for the next transferred patient.

This method balances the transferred and control groups according to the admission time which is important given the decreasing case fatality observed during the period. ${ }^{7}$ In some centres where a large proportion of patients have been transferred, the method will result in the inclusion of all non-transferred patients as controls over the study period. It is expected that in instances when several patients have been transferred the same day from the same hospital, the pool of eligible control patients might not be large enough to attain the ratio of 4 controls per transfer. The maximum number of participants enrolled in the cohort is therefore of 3305 including 661 transferred and 2644 control patients.

\section{Data and materials}

The TRANSCOV cohort database will integrate clinical and medico-administrative data (see figure 1). ICU and emergency teams will be invited to fill in the relevant electronic case report forms (CRF) for each patient. The CRFs will gather information regarding the initial ICU stay (CRF_origin and CRF_control), the transfer (CRF transfer) and the ICU stay in the destination hospital (CRF_destination). A specifically designed unique identifier (id_transcov) will allow linkage between the three CRFs of transferred patients.

Items of information in the CRFs will cover sociodemographic, clinical, biological and treatment characteristics, such as key haemodynamic, respiratory and therapeutic parameters, as well as severity scores (SOFA (Sequential Organ Failure Assessment Score) and SAPS II(Simplified Acute Physiology Score)). ${ }^{89}$ Items in the 'CRF_transfer' will identify the number of vectors used (eg, ambulance, train, then ambulance), the number of changes in clinical teams involved (medical offload) and the number of changes of equipment (eg, ventilators, material offload).
Medical and material offloads represent likely determinants of the clinical outcome of the transfer.

Each centre will fill in electronic version of the CRFs using the Ennov Clinical data collection software. An English-language version of the CRF destination is produced to be used by the foreign healthcare teams who have received patients abroad. To ensure good quality of data, range of credible values, consistency checks and audit trails for data modifications are implemented in Ennov Clinical and are described in a support document provided to all investigators. Electronic data entry is protected thanks to a secure connection to the Rennes University Hospital server. Individual identifiers will be provided to each user, such that the access is limited to their centre's data only. Ultimately, each investigator is responsible for the accuracy, quality and relevance of all data entered. Recruitment of centres and enrolment of patients in the study has started in January 2021 and is expected to finish in December 2021.

Clinical data will be enriched through data linkage to the 'Système National des Données de Santé' (SNDS). ${ }^{10}$ The SNDS is a medicoadministrative database gathering all information related to the consumption of hospital and ambulatory care (coded with ICD 10 for diagnosis, and ATC for treatment) of virtually all French citizens $(98 \%)$. SNDS data will allow identification of relevant comorbidities and prior prognostic factors (eg, arterial hypertension, cardiovascular or kidney diseases) based on healthcare contacts and prescriptions in the preceding year. Moreover, the entire care pathway of patients can be constructed from SNDS including initial hospitalisation, admission/successive admission to the ICU and destination after discharge (rehabilitation care, home, death). We intend to follow patients up to 1 year after the date of the hospital admission for COVID-19. The objective of this follow-up is to compare use of healthcare resources (eg, respiratory support, oxygen, physiotherapy) and 
survival beyond acute care in both transferred and control groups. Particular attention will be paid to the consumption of psychotropic drugs suggestive of post-traumatic stress disorder treatment. For all participants, origin hospitals will collect the 'numéro d'identification au répertoire' (NIR), a unique identifier used by the French social security services. With the NIR, deterministic data linkage can be performed between clinical (CRF) and SNDS data ensuring better precision and completeness than probabilistic linkage. The completeness criterion is particularly important for TRANSCOV given the relatively small number of transferred patients. Double entry of the NIR will be requested to limit transcription errors.

\section{Statistical analysis}

After linking the clinical and SNDS data, a quality control of the database will be carried out, looking particularly for aberrant or missing data. In the event of missing data in the CRF, multiple imputation methods will be considered and used if the conditions of application are met (random missing data). Algorithms validated by the 'REDSIAM network' ${ }^{11}$ will be used to generate variables identifying specific co-morbidities from the SNDS data. A descriptive analysis of the study population will be conducted with the usual parameters (mean $\pm \mathrm{SD}$, median, min, max for the quantitative variables, number (\%) for the qualitative variables). Data relating to the modalities of transfer (vectors, duration of transport, medical and material offloads ...) will also be described for the group concerned. Comparative analysis will first focus on the modalities and vectors used during the transfers. The main objectives of the study, however, are the comparisons between transferred and control patients. Comparisons will be made for all data collected up to the date of transfer with the appropriate statistical tests (including Student and $\chi^{2}$ ). With regard to the primary endpoint (survival) and secondary endpoints, the aim of the analysis will be to make the groups as comparable as possible on prognostic factors in order to identify in an unbiased way the impact of the transfer. An analysis with a propensity score (inverse probability of treatment weighting) and multivariate regression modelling will be carried out for this purpose. We will use Cox model for 'time-to-event' events, linear regression for quantitative data and logistic regression for binary events. For survival models, the date of origin will be the date of hospitalisation for COVID-19.

\section{DISCUSSION}

An important feature of the TRANSCOV cohort is the high number of centres involved in the study since more than 230 hospitals are invited to take part. At a time of the clinical and research workloads associated to COVID-19 remain very high, ensuring participation of such a large number of clinical teams is a real challenge. Two steps have been taken in order to maximise success in that matter. The first one consisted in establishing early on (June 2020) a steering comity which involves representatives from all French emergency and critical care scientific societies. The role of the comity is primarily to supervise the conception and implementation of the study. It is also is key means of communication and motivation of the clinicians. Second, the team of investigators has endeavoured to minimise the workload of collaborating centres by restricting the collection of clinical data to an essential set of information complementary to that available in medicoadministrative database. In a pilot phase of the CRF, time to retrieve clinical data from medical records was estimated at around $30 \mathrm{~min}$ per patient.

The TRANSCOV cohort is one of the several components of the wider TRANSCOV project that aims at drawing all lessons from what was a unique experience of inter-regional and international solidarity. The project includes thus both a qualitative and an economic evaluation. In many ways, the spring 2020 wave of COVID-19 patient's transfers represents an organisational innovation. Relying on interviews with members of the clinical teams, representative from the regional health authorities and of the ministry of health, the qualitative component of the TRANSCOV project is exploring how this was made possible. The economic evaluation will compare costs according to transfer modalities. As mentioned earlier, a specific clinical study is being designed for exploring medium term psychological consequences of distant transfers.

At the time of writing this article, early initial reports based on a sample of transferred patients, ${ }^{12}$ and on surveillance data (personal communication, Marc Antoine Sanchez), suggest that long-distance transfers did not result in increased short-term mortality risk for the COVID-19 patients involved. These results support the assumption that in spring 2020 ICU teams operated a careful selection of eligible patients for transfer. The TRANSCOV Cohort will provide ample opportunities to describe in details the process of patients selection, and to compare short and medium outcomes according to transfer status and vectors used.

\section{Ethics and dissemination}

The study was approved by the independent Ethical and Scientific Committee: CESREES on the 16 July 2020 (file no. 2046524). For patients whose entire care pathway took place in France, investigating origin hospitals will send an individual information notice that clearly explains them or their legal guardian the objectives and procedures of the study, as well as how to decline participation (see online supplemental appendix). For patients transferred abroad, the consent procedure will be adapted in order to comply with the relevant country specific patient consent legislation.

Results will be published as scientific articles in peerreviewed biomedical journals and presented in national and international conferences. A TRANSCOV authors list will be used to acknowledge the contribution of all named collaborators who took part to data collection. The TRANSCOV steering comity will endorse the detailed 
authorship of articles transcribing the study main results. The steering comity includes representatives from French scientific societies in intensive care, emergency medicine and infectious diseases as well as the medical service of the army.

\section{Patient and public involvement}

There were no patients' involvement in the research question development or in the design of the study. In order to disseminate results to patients, a summarised version written in non-technical language will be posted on the principal investigator website and will be sent to all collaborating centres.

\section{Author affiliations}

${ }^{1}$ Univ Rennes, EHESP, CNRS, ARENES - UMR 6051, Rennes, France

${ }^{2}$ Service de Médecine Intensive Réanimation, CHU Brest, Brest, France

${ }^{3}$ Université Pierre et Marie Curie Faculté de Médecine, Paris, France

${ }^{4}$ Anesthesiology and Critical Care, Hopitaux Universitaires de Strasbourg, Strasbourg, France

${ }^{5}$ Direction centrale du service de santé des armées, Paris, France

${ }^{6} \mathrm{CHR}$ Metz-Thionville, Metz, France

${ }^{7}$ Emergency Department SAMU-SMUR 95, CHG Pontoise-Beaumont/Oise Pontoise,

Pontoise, France

${ }^{8}$ Cellule d'Urgence Médico-Psychologique (CUMP), Centre Régional du

Psychotraumatisme (CRP), Hôpital Edouard Herriot, HCL, Lyon, Auvergne-Rhône-

Alpes, France

${ }^{9}$ France Ministère des Solidarités et de la Santé Direction Générale de la Santé, Paris, France

${ }^{10}$ Univ Rennes, EHESP, REPERES (Recherche en pharmaco-épidémiologie et recours aux soins) - EA 7449, Rennes, France, Rennes, France

Acknowledgements The authors would like to thank Kaldoun Kuteifan, Pierre Fillatre, Guillaume Louis and Arnaud Romoli for taking part to the piloting of the CRF. Useful comments were gathered on an initial version of the protocol during a session of the Strategic Comity (COMSTRAT) that supervises the overall TRANSCOV project. The expertise of the clinical research team of the Rennes University Hospital has proved invaluable for setting up and implementing the data collection process. In this context, special thanks go to Kristell Coat (project manager), Mathilde Leonard (data manager), Alain Renault and Loïc Fin.

Contributors $\mathrm{OG}, \mathrm{SB}$ and EL conceived and wrote the initial version of the study protocol. AR, EM, JP, FB, J-MP, AR-H, NP and CF commented on successive versions of the protocol and contributed to the design of the CRFs. OG wrote the article whose final version was approved by all authors.

Funding This work is supported by the Directorate General of Health (DGS) of the French Ministry of Health. Grant / Award number : N/A

Competing interests None declared.

Patient and public involvement Patients and/or the public were involved in the design, or conduct, or reporting, or dissemination plans of this research. Refer to the Methods section for further details.

Patient consent for publication Not applicable.

Provenance and peer review Not commissioned; externally peer reviewed.
Supplemental material This content has been supplied by the author(s). It has not been vetted by BMJ Publishing Group Limited (BMJ) and may not have been peer-reviewed. Any opinions or recommendations discussed are solely those of the author(s) and are not endorsed by BMJ. BMJ disclaims all liability and responsibility arising from any reliance placed on the content. Where the content includes any translated material, BMJ does not warrant the accuracy and reliability of the translations (including but not limited to local regulations, clinical guidelines, terminology, drug names and drug dosages), and is not responsible for any error and/or omissions arising from translation and adaptation or otherwise.

Open access This is an open access article distributed in accordance with the Creative Commons Attribution Non Commercial (CC BY-NC 4.0) license, which permits others to distribute, remix, adapt, build upon this work non-commercially, and license their derivative works on different terms, provided the original work is properly cited, appropriate credit is given, any changes made indicated, and the use is non-commercial. See: http://creativecommons.org/licenses/by-nc/4.0/.

ORCID iD

Olivier Grimaud http://orcid.org/0000-0003-1087-9197

\section{REFERENCES}

1 Suivi du Covid-19 en France. Available: https://mapthenews.maps. arcgis.com/apps/opsdashboard/index.html\#/5df19abcf8714bc590a3 b143e14a548c [Accessed 02 Jul 2020].

2 Hong TH, Lee H, Jung JJ, et al. Inter-facility transport on extracorporeal life support: clinical outcomes and comparative analysis with in-house patients. Korean J Thorac Cardiovasc Surg 2017;50:363-70.

3 Flabouris A, Hart GK, George C. Outcomes of patients admitted to tertiary intensive care units after interhospital transfer: comparison with patients admitted from emergency departments. Crit Care Resusc 2008;10:97-105.

4 Sammito S, Turc J, Post J, et al. Analysis of European air medical evacuation flights of coronavirus disease 2019 patients. Air Med J 2021;40:211-5.

5 Sammito S, Post J, Ritter DM, et al. Innereuropäische aeromedicalevacuation-transporte Im rahmen von COVID-19. Der Notarzt 2020;36:263-70.

6 The BMJ. Chapter 8. case-control and cross sectional studies. In: The BMJ: leading general medical Journal. research. education. Comment, 2020. https://www.bmj.com/about-bmj/resourcesreaders/publications/epidemiology-uninitiated/8-case-control-andcross-sectional

7 Piroth L, Cottenet J, Mariet A-S, et al. Comparison of the characteristics, morbidity, and mortality of COVID-19 and seasonal influenza: a nationwide, population-based retrospective cohort study. Lancet Respir Med 2021;9:S2213260020305270.

8 Vincent J-L, Moreno R, Takala J, et al. The sofa (sepsis-related organ failure assessment) score to describe organ dysfunction/failure. Intensive Care Med 1996;22:707-10.

9 Le Gall JR, Lemeshow S, Saulnier F. A new simplified acute physiology score (SAPs II) based on a European/North American multicenter study. JAMA 1993;270:2957-63.

10 Scailteux L-M, Droitcourt C, Balusson F, et al. French administrative health care database (SNDS): the value of its enrichment. Therapie 2019;74:215-23.

11 Goldberg M, Carton M, Doussin A, et al. [The redsiam network]. Rev Epidemiol Sante Publique 2017;65 Suppl 4:S144-8.

12 Painvin B, Messet $\mathrm{H}$, Rodriguez M, et al. Inter-hospital transport of critically ill patients to manage the intensive care unit surge during the COVID-19 pandemic in France. Ann Intensive Care 2021;11:54. 